\title{
let-7b suppresses apoptosis and autophagy of human mesenchymal stem cells transplanted into ischemia/reperfusion injured heart 7by targeting caspase-3
}

\author{
Onju Ham ${ }^{1 \dagger}$, Se-Yeon Lee ${ }^{1 \dagger}$, Chang Youn Lee ${ }^{2 \dagger}$, Jun-Hee Park ${ }^{2}$, Jiyun Lee ${ }^{3}$, Hyang-Hee Seo ${ }^{3}$, Min-Ji Cha ${ }^{1,4}$,
} Eunhyun Choi ${ }^{1,4}$, Soonhag Kim ${ }^{1,4}$ and Ki-Chul Hwang ${ }^{1,4^{*}}$

\begin{abstract}
Introduction: Mesenchymal stem cells (MSCs) have therapeutic potential for the repair of myocardial injury. The efficacy of MSC therapy for myocardial regeneration mainly depends on the survival of cells after transplantation into the infarcted heart. In the transplanted regions, reactive oxygen species (ROS) can cause cell death, and this process depends on caspase activation and autophagosome formation.

Methods: A Software TargetScan was utilized to search for microRNAs (miRNAs) that target caspase-3 mRNA. Six candidate miRNAs including let-7b were selected and transfected into human MSCs in vitro. Expression of MEK-EKR signal pathways and autophagy-related genes were detected. Using ischemia/reperfusion model (I/R), the effect of MSCs enriched with let-7b was determined after transplantation into infarcted heart area. Miller catheter was used to evaluate cardiac function.

Results: Here, we report that let-7b targets caspase-3 to regulate apoptosis and autophagy in MSCs exposed to ROS. Let-7b-transfected MSCs (let-7b-MSCs) showed high expression of survival-related proteins, including p-MEK, p-ERK and BCl-2, leading to a decrease in Annexin V/PI- and TUNEL-positive cells under ROS-rich conditions. Moreover, autophagy-related genes, including Atg5, Atg7, Atg12 and beclin-1, were significantly downregulated in let-7b-MSCs. Using a rat model of acute myocardial infarction, we found that intramyocardial injection of let-7b-MSCs markedly enhanced left ventricular (LV) function and microvessel density, in accordance with a reduced infarct size and the expression of caspase-3.
\end{abstract}

Conclusions: Taken together, these data indicate that let-7b may protect MSCs implanted into infarcted myocardium from apoptosis and autophagy by directly targeting caspase-3 signaling.

\section{Introduction}

Despite continuous improvements in the prevention and treatment of heart disease, ischemic heart disease is the most common cause of mortality worldwide [1]. Ischemic injury to the heart causes various types of cardiomyocyte

\footnotetext{
*Correspondence: kchwang@cku.ac.kr

${ }^{\dagger}$ Equal contributors

'Catholic Kwandong University International St. Mary's Hospital, Incheon Metropolitan City 404-834, Republic of Korea

${ }^{4}$ Institute for Bio-Medical Convergence, College of Medicine, Catholic Kwandong University, Gangneung-si, Gangwon-do 210-701, Republic of Korea

Full list of author information is available at the end of the article
}

death such as necrosis, apoptosis, or autophagy. Various treatment methods for ischemic heart disease have been explored, including gene-based, growth factor-based, and cell-based therapies [2]. Mesenchymal stem cells (MSCs) are an attractive source for cell-based therapies and can be used to repair bone [3], cartilage [4], and heart tissue [5]. In fact, over the last decade, transplantation of MSCs has been considered as a therapeutic method for repairing the infarcted region of ischemic hearts [1]. However, the low viability of cells after transplantation has hampered therapeutic efficacy of cell therapy. Thus, identifying apoptosis-related factors and promoting the survival of

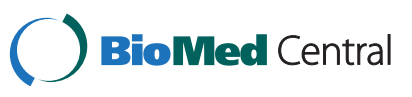

(c) 2015 Ham et al. Open Access This article is distributed under the terms of the Creative Commons Attribution 4.0 International License (http://creativecommons.org/licenses/by/4.0/), which permits unrestricted use, distribution, and reproduction in any medium, provided you give appropriate credit to the original author(s) and the source, provide a link to the Creative Commons license, and indicate if changes were made. The Creative Commons Public Domain Dedication waiver (http://creativecommons.org/publicdomain/zero/1.0/) applies to the data made available in this article, unless otherwise stated. 
transplanted MSCs are key goals for improving the utilization of these cells in cell therapy.

Reactive oxygen species (ROS) play an important role as an inducer of cell death pathways, including apoptosis, anoikis, and autophagy, in ischemic hearts [6]. Among these cell death pathways, apoptosis plays an important role in the elimination of unnecessary cells and is induced by the orchestrated activity of caspase family proteins [7]. Caspase family proteins play a critical role in both the intrinsic and extrinsic apoptosis pathways. During apoptosis, caspases initiate a two-step pathway in which initiator caspases (caspase-8, caspase-9, and caspase-10) cleave executioner caspases (caspase- 3 and caspase-7) to activate them [8]. Both caspase- 8 and caspase- 9 activate caspase- 3 , which in turn cleaves several crucial substrates including the DNA repair enzyme poly(ADP-ribose) polymerase (PARP) [9]. Autophagy, or self-digestion, is also activated in response to stressors such as ischemia/reperfusion (I/R). Substrates, such as aggregated proteins or damaged organelles, are engulfed during autophagy to form autophagosomes [10, 11]. Although autophagy plays a protective role under a physiologic condition by degrading damaged mitochondria and protein aggregates, excessive autophagy under pathologic conditions can lead to organ dysfunction by degrading essential proteins and organelles [6].

MicroRNAs (miRNAs) are small, noncoding, regulatory RNAs composed of 19-22 nucleotides [12]. miRNAs can act post-transcriptionally to either target specific mRNAs for degradation or suppress their translation [12, 13]. Consequently, miRNAs play critical roles in the regulation of multiple biological processes, including development, organogenesis, cell proliferation, cell differentiation, and apoptosis [13]. However, little is known about how miRNAs regulate cell survival. Recent studies have successfully established a functional link between cell survival and a discrete group of survival-regulating miRNAs, including miRNA-1 [14], miRNA-125 [15], miRNA-206 [14], miRNA-210 [16, 17] and miRNA-708 [18]. In addition, miRNA-210 regulates survival via a positive feedback loop during hypoxia [16]. Nevertheless, most studies have focused on cancer cells as a model to study the mechanisms of hypoxia-regulated miRNAs in their endeavor to escape death in the ischemic microenvironment of tumors, and the role of miRNAs in the survival of transplanted MSCs for therapeutic purposes remains largely unknown. Therefore, in the present study, we examined the feasibility of enhancing survival of transplanted MSCs by modulating miRNA that targets key apoptotic molecule caspase-3. We observed that cleaved (activated) caspase and PARP increased after exposure to ROS in MSCs. Furthermore, our data showed that let-7b plays an important role in cell survival, apoptosis, and autophagy in MSCs under oxidative stress. The results of our study suggest that miRNA-mediated fortification of MSCs by enhancing cell survival can be a potential therapeutic approach to treat infarcted heart.

\section{Materials and methods Culture of human MSCs}

Human bone marrow-derived mesenchymal stem cells (hMSCs) were purchased from Lonza (Walkersville, MD, USA). hMSCs were cultured according to the manufacturer's instructions. We used hMSCs at passages 7-10 for experiments, and the cells were cultured in low-glucose Dulbecco's modified Eagle's medium (DMEM; Life Technologies Corporation, Grand Island, NY, USA) containing $10 \%$ fetal bovine serum (FBS; Life Technologies Corporation).

\section{miRNA mimic transfection}

The miRNA mimics, which are synthesized mature miRNAs or negative control miRNAs with random sequence (NC), were purchased from Genolution Pharmaceuticals (Genolution Inc., Seoul, Korea). Either miRNA mimic or NC (100 nM each) was transfected into hMSCs using siLentFect ${ }^{\text {tix }}$ Lipid Reagent (Bio-Rad, Hercules, CA, USA) according to the manufacturer's instructions. After 4 hours of transfection, the medium was changed to $10 \%$ FBS-containing DMEM with $1 \%$ antibiotics.

\section{Treatment of cells with hydrogen peroxide}

The cells were incubated in low-glucose DMEM containing $10 \%$ FBS. Various concentrations $(100-750 \mu \mathrm{M})$ of hydrogen peroxide $\left(\mathrm{H}_{2} \mathrm{O}_{2}\right.$; Sigma, St. Louis, MO, USA) were then added to the medium and incubated with the cells for 6 hours.

\section{Cell viability assay}

Cell viability was determined using a WST-8 (2-(2-methoxy-4-nitrophenyl)-3-(4-nitrophenyl)-5-(2,4-disulfophenyl)-2H-tetrazolium) assay kit (CCK-8 assay kit; Dojindo, Kumamoto, Japan). hMSCs were seeded in 96-well plates (Corning Incorporated, Corning, NY, USA) at a density of $5 \times 10^{3}$ cells per well. The cells were transfected with 100 $\mathrm{nM}$ let-7b mimics for 4 hours. After transfection for 4 hours, the medium was changed to fresh $10 \%$ DMEM and the cells were incubated for 24 hours. After incubation, cells were treated with varying concentrations of $\mathrm{H}_{2} \mathrm{O}_{2}$ for 6 hours in $10 \%$ DMEM. The cells were then washed twice with medium, and $100 \mu \mathrm{l}$ CCK- 8 reagent was added to each well. The samples were subsequently incubated for 2 hours at $37{ }^{\circ} \mathrm{C}$. The absorbance of the samples was measured at $450 \mathrm{~nm}$ against a background control using a microplate reader (Bio-Rad).

\section{Real-time PCR}

MSCs were plated at a density of $1.5 \times 10^{5}$ cells per well using six-well plates. The cells were first transfected with 
$100 \mathrm{nM}$ let-7b mimics as described above, and then exposed to varying concentrations of $\mathrm{H}_{2} \mathrm{O}_{2}$ for 6 hours in $10 \%$ DMEM. Total RNA was prepared using Trizol $^{\circ}$ reagent (Sigma). cDNA for real-time PCR was prepared from purified total RNA using reverse transcriptase (Taqman ${ }^{\circ}$ MicroRNA Reverse Transcriptase Kit; Applied Biosystems, Carlsbad, CA, USA) with specific miRNA primers. U6 served as a control. The following protocol was used for amplification: $95{ }^{\circ} \mathrm{C}$ for 10 minutes, followed by 40 cycles of $95{ }^{\circ} \mathrm{C}$ for 15 seconds and $60{ }^{\circ} \mathrm{C}$ for 60 seconds. The sequence of the human origin let-7b (hsa-let-7b) primer was 5' -UUG GUG UGU UGG AUG AUG GAG U-3'.

For detecting autophagy-related genes, cDNA was synthesized using a Reverse Transcription System (Promega Corporation, Fitchburg, WI, USA) according to the manufacturer's instructions. One microgram of RNA was reverse-transcribed in a $20 \mu \mathrm{l}$ reaction $(5 \mathrm{mM}$ $\mathrm{MgCl}_{2}, 10 \mathrm{mM}$ Tris- $\mathrm{HCl}\left(\mathrm{pH} 9.0\right.$ at $\left.25{ }^{\circ} \mathrm{C}\right), 50 \mathrm{mM}$ $\mathrm{KCl}, 0.1 \%$ Triton X-100, $1 \mathrm{mM}$ dNTP, $20 \mathrm{U}$ RNase inhibitor, $0.5 \mu \mathrm{g}$ oligo $(\mathrm{dT})$ primer, and $10 \mathrm{U}$ reverse transcriptase) for 15 minutes at $42{ }^{\circ} \mathrm{C}$, and then ended at $99{ }^{\circ} \mathrm{C}$ for 5 minutes. PCR primers were synthesized by Bioneer Corporation (Daejeon, Korea), and the primers used were as follows: GAPDH, 5'-CAT GGG TGT GAA CCA TGA GAA-3' and 5'-GGT CAT GAG TCC TTC CAC GAT-3' (133 base pairs (bp)); autophagy-related (ATG) 5, 5' -AGC AAC TCT GGA TGG GAT TG-3' and 5'-AGG TCT TTC AGT CGT TGT CTG-3' (139 bp); ATG7, 5' -TTT TGC TAT CCT GCC CTC TG-3' and '5GCT GTG ACT CCT TCT GTT TGAC-3' (142 bp); ATG12, 5' -ACC ATC CAA GGA CTC ATT GAC-3' and 5'-CCA TCA CTG CCA AAA CAC TC-3' (142 bp); and beclin 1, 5' -AAG AGG TTG AGA AAG GCG AG-3' and 5' -TGG GTT TTG ATG GAA TAG GAG C-3' (111 bp). PCR reactions were run on a LightCycler 480 SYBR Green I Master (Roche, Penzberg, Germany) protocol. PCR conditions were set to $95{ }^{\circ} \mathrm{C}$ for 3 minutes; $95{ }^{\circ} \mathrm{C}$ for $30 \mathrm{sec}-$ onds with 40 cycles of denaturation; $55^{\circ} \mathrm{C}$ for 30 seconds; $72{ }^{\circ} \mathrm{C}$ for 90 seconds; and $72{ }^{\circ} \mathrm{C}$ for 10 minutes as the final extension. The threshold cycle $(\mathrm{Ct})$ of each target gene, which was located in the linear amplification phase of the PCR, was measured automatically and normalized to the cycle number of control (U6 and GAPDH for miRNAs and mRNAs, respectively). The relative expression levels of each miRNA or mRNA were measured $\left({ }^{\Delta \Delta} \mathrm{Ct}\right)$ and reported as fold induction $\left({ }^{2 \Delta \Delta} \mathrm{Ct}\right)$.

\section{Western blotting analysis}

hMSCs were plated at a density of $3 \times 10^{5}$ per $60 \mathrm{~mm}$ dish. The cells were first transfected with $100 \mathrm{nM}$ let-7b mimics as described above, and then exposed to varying concentrations of $\mathrm{H}_{2} \mathrm{O}_{2}$ for 6 hours in 10 \% DMEM. hMSCs were lysed in $1 \times$ lysis buffer (Cell Signaling
Technology, Beverly, MA, USA) with protease inhibitor (Roche, Basel, Switzerland) and phosphatase inhibitor cocktail (Roche) at $4{ }^{\circ} \mathrm{C}$ for 25 minutes. The protein concentrations were determined using a BCA assay (Pierce Biotechnology, Rockford, IL, USA). Equal amount of proteins were subjected to 8 or $10 \%$ SDS-PAGE. Proteins were transferred to polyvinylidene difluoride membranes (PVDF; Millipore, Billerica, MA, USA) at $100 \mathrm{~V}$ and $135 \mathrm{~mA}$ for 100 minutes. The membranes were blocked with Tris-buffered saline- $0.1 \%$ Tween 20 (TBS-T; both Sigma) and $10 \%$ skim milk (BD Science, San Jose, CA, USA) for 1 hour at room temperature or overnight at $4{ }^{\circ} \mathrm{C}$. The blots were then incubated with primary antiPARP, anti-caspase-3, anti-phospho-mitogen-activated protein kinase (MEK), anti-MEK, anti-p-extracellular signal regulated kinase (ERK), anti-ERK, anti-Bcl-2, anti-Bax, anti-light chain $3 \mathrm{~A} / \mathrm{B}$ (LC3A/B), and anti- $\beta$ actin antibodies for 1 hour at room temperature or overnight at $4{ }^{\circ} \mathrm{C}$. Polyclonal anti-phospho-MEK, antiMEK, anti-ERK, anti-LC-3IIA/B, and anti-PARP antibodies were obtained from Cell Signaling Technology. Monoclonal anti-phospho-ERK and monoclonal anti$\mathrm{Bcl} 2$ antibodies were obtained from Santa Cruz Biotechnology (Santa Cruz, CA, USA), polyclonal anti-Bax antibody was obtained from Enzo Life Sciences (Ann Arbor, MI, USA), polyclonal anti-caspase-3 antibody was obtained from Millipore, and $\beta$-actin antibody was obtained from Sigma. All antibodies were diluted 1:1000 with TBS-T and $5 \%$ nonfat dried milk. The membranes were then washed three times in $1 \times$ TBS-T at room temperature and incubated with a horseradish peroxidaseconjugated rabbit or mouse secondary antibody (Santa Cruz Biotechnology). After washing the membrane six times, immunoreactive proteins were detected using an ECL system (Amersham Biosciences, Tokyo, Japan). The images were quantified using ImageJ software.

\section{Annexin V/propidium iodide staining}

hMSCs were cultured in four-well culture dishes $(1 \times$ $10^{4}$ cells per well; Corning Incorporated). The cells were treated with $500 \mu \mathrm{M} \mathrm{H}_{2} \mathrm{O}_{2}$ for 6 hours with or without prior let-7b transfection. After 6 hours of $\mathrm{H}_{2} \mathrm{O}_{2}$ treatment, the cells were washed with ice-cold phosphatebuffered saline (PBS; Life Technologies Corporation) for 5 minutes and fixed with $4 \%$ formalin (Sigma) for $10 \mathrm{mi}$ nutes. After blocking with $500 \mu \mathrm{l}$ Annexin-binding buffer, the cells were stained with Annexin V-fluorescein isothiocyanate (FITC) at room temperature in the dark for 15 minutes. The dishes were washed with ice-cold PBS, stained with propidium iodide Annexin V/PI staining (PI), and diluted with Annexin-binding buffer at room temperature in the dark for 5 minutes. All images of Annexin V/PI-positive cells were detected by laser scanning confocal microscopy (LSM 700; Carl Zeiss, 
Thornwood, NY USA), and the images were transferred to a computer equipped with ZEN Lite (Carl Zeiss).

\section{Measurement of caspase-3 activity}

Relative caspase- 3 activity was determined using a caspase3 activity assay kit (Roche). In brief, the cultured MSCs (2 $\times 10^{6}$ ) were lysed in $1 \times$ DTT (USB Corporation, Cleveland, $\mathrm{OH}, \mathrm{USA}$ ) for 1 minute. Lysates were collected and loaded onto a plate coated with monoclonal caspase- 3 antibody. Upon substrate cleavage, free fluorescent AFC (7-amino-4trifluoromethylcoumarine) was quantified using a microplate reader (Bio-Rad).

\section{Terminal deoxynucleotidyltransferase-mediated dUTP nick-end labeling assay}

A terminal deoxynucleotidyltransferase-mediated dUTP nick-end labeling (TUNEL) assay was performed according to the manufacturer's instructions (Millipore). hMSCs were plated in a four-well culture dish $\left(1 \times 10^{4}\right.$ cells per well) and treated with $500 \mu \mathrm{M} \mathrm{H}_{2} \mathrm{O}_{2}$ for 6 hours with or without prior let-7b transfection. After the slides were rinsed with PBS, the cells were fixed with $10 \%$ formaldehyde (Sigma) for 10 minutes. The slides were treated with $3.0 \% \mathrm{H}_{2} \mathrm{O}_{2}$ and TdT enzyme for 1 hour followed by digoxygenin-conjugated nucleotide substrate at $37{ }^{\circ} \mathrm{C}$ for 30 minutes. Nuclei were stained with 3,3' -diaminobenzidine (DAB; Vector Laboratories, Burlingame, CA, USA) for 5 minutes, and the slides were counterstained with methyl green (Sigma). Dark-brown-stained nuclei indicated apoptotic cells. The slides were observed by a virtual microscopy (BX51/dot Slide; Olympus, Tokyo, Japan).

\section{Luciferase assay}

The miRNAs targeting caspase-3 were screened based on the TargetScan miRNA-target prediction database [19]. We amplified the 959 base pair 3' untranslated region (UTR) of human caspase-3, which contains the binding sites for seven different candidate miRNAs, and the $3^{\prime}$ UTR of human caspase- 3 was cloned into the pmirGLO vector (Promega Corporation, Fitchburg, WI, USA). HeLa cells (ATCC, Rockville, MD, USA) were plated in 24-well plates (Corning Incorporated) at a density of $2 \times 10^{4}$ cells per well. The pmirGLO vector containing the 3' UTR of caspase-3 was co-transfected with let-7b mimic or NC using siLentFect ${ }^{\text {mit }}$ (Bio-Rad). Luciferase activity was measured 48 hours later using a luminometer and the Dual Luciferase Assay (Promega Corporation) according to the manufacturer's instructions. Renilla luciferase was used to normalize the cell number and transfection efficiency.

\section{Design of a miRNA-detecting molecular beacon}

We have previously developed a molecular beacon (MB) to detect miRNA expression in single cells [20]. MBs are oligonucleotide hybridization probes that indicate the presence of specific nucleic acids. We designed a MB to detect the presence of let-7b, which forms a partially double-stranded structure with a longer $\mathrm{Cy} 3$ modified sequence (5'-AAC CAC ACA ACC TAC TAC CTC A-3'Cy3) and a black hole quencher dye 1 (BHQ1) modified shorter sequence (3'-TGA TGG AGT-5'-BHQ1). The longer sequence of the $\mathrm{MB}$ was designed to complementarily match the sequence of let-7b (5'-TGA GGT AGT AGG TTG TGT GGT T-3') so that when MB and let-7b bind to each other, making the fluorophore and the quencher sufficiently separated, fluorescence from the fluorophore can be detected. These oligonucleotides used for the MB were manufactured by Bioneer Corporation. To find the working concentration of the $\mathrm{MB}$, we conducted an invitro assay. Varying concentrations of $\mathrm{MB}(0,1,5,10,20$, 50 , and $100 \mathrm{pM}$ ) were reacted with $100 \mathrm{nM}$ let-7b mimic in Eppendorf tubes for 1 hour at $37^{\circ} \mathrm{C}$, and the fluorescence intensity of each group was measured (Varioskan Flash; Thermo Scientific, Waltham, MA, USA). miRNA23 has a completely irrelevant sequence to the let-7b, and thus a MB designed to detect miR-23 served as a NC. Since a denatured $\mathrm{MB}$ should emit fluorescence, boiled $\mathrm{MB}$ (at $95^{\circ} \mathrm{C}$ for 10 minutes) served as a positive control.

\section{Detection of let-7b using a MB}

hMSCs were seeded onto 24-well plates at a density of 2 $\times 10^{4}$ cells per well. After transfection of $100 \mathrm{nM}$ let-7b mimic, the cells were incubated with or without $500 \mu \mathrm{M}$ $\mathrm{H}_{2} \mathrm{O}_{2}$. To detect cellular let-7b, the cells were transfected with $50 \mathrm{pM}$ MB. The fluorescence intensity was measured (Varioskan Flash; Thermo Scientific).

\section{$\mathrm{I} / \mathrm{R}$ injury and transplantation of MSCs}

$\mathrm{I} / \mathrm{R}$ injury was induced in male Sprague-Dawley rats (250 \pm 30 g; Coretech, Pyeongtaek, Korea) by surgical occlusion of the left anterior descending coronary artery according to previously described procedures [21]. For transplantation, $1 \times 10^{6}$ cells were suspended in $30 \mu \mathrm{l}$ PBS and injected from the injured region to the border using a Hamilton syringe with a 30 -gauge needle. Throughout the operation, the animals were ventilated with $95 \% \mathrm{O}_{2}$ and $5 \% \mathrm{CO}_{2}$ using a Harvard ventilator (Harvard Apparatus, Holliston, MA, USA). Five animals per group (ligation, NC-MSCs, let-7b-MSCs) were used for morphological and functional analysis.

Left ventricular catheterization for hemodynamic analysis Left ventricular catheterization was performed 3 weeks after infarction to assess hemodynamics. A Millar Mikrotip $2 \mathrm{~F}$ pressure-volume transducer (model SPR-838; Millar Instruments, Houston, TX, USA) was introduced into the left ventricle via the right carotid artery under anesthesia. All data were analyzed offline with PVAN 3.5 software (Millar Instruments). 


\section{Histological analysis and determination of fibrosis area}

Heart tissues were fixed in $3.7 \%$ buffered formaldehyde and embedded in paraffin. Tissue sections $(5 \mu \mathrm{m}$ thickness) were deparaffinized, dehydrated, and rinsed with PBS. Fibrosis was analyzed by Masson's trichrome staining kit (Sigma). Antigen retrieval was performed with $10 \mathrm{mM}$ sodium citrate ( $\mathrm{pH}$ 6.0; Sigma) in a microwave for 10 minutes. The sections were incubated in $3 \%$ $\mathrm{H}_{2} \mathrm{O}_{2}$ to quench endogenous peroxidase activity. The samples were blocked in $2.5 \%$ normal horse serum (Sigma) and subsequently incubated with antibodies including anti-mouse CD31 and anti-rabbit caspase-3. All antibodies were diluted 1:500 with $1.5 \%$ normal horse serum. All antibodies were obtained from Santa Cruz Biotechnology. FITC-conjugated goat anti-mouse IgG (Jackson ImmunoResearch Laboratories, West Grove, PA, USA) and rhodamine-conjugated goat anti-rabbit IgG (Jackson ImmunoResearch Laboratories) were used as secondary antibodies. All images of CD31 and caspase-3 were obtained using laser scanning confocal microscopy (LSM 710; Carl Zeiss, Thornwood, NY, USA) and transferred to a computer equipped with Zen Light Edition (Zeiss) for analysis. The areas are expressed as percentages of the total left ventricle. Fibrosis was also analyzed by Masson's trichrome staining.

\section{Experimental ethics policy}

All experimental procedures for animal studies were approved by the Committee for the Care and Use of Laboratory Animals at Yonsei University College of Medicine and were performed in accordance with the Committee's Guidelines and Regulations for Animal Care.

\section{Statistical analysis}

Data are expressed as the mean \pm standard error of the mean of at least three independent experiments. Comparisons between more than two groups were performed by one-way analysis of variance using Bonferroni's correction. $P$ value of less than 0.05 was considered significant.

\section{Results}

\section{$\mathrm{H}_{2} \mathrm{O}_{2}$-induced apoptosis of hMSCs}

We used $\mathrm{H}_{2} \mathrm{O}_{2}$ to simulate ROS-mediated cell death in our experiments. To induce apoptosis, hMSCs were treated with varying concentrations of $\mathrm{H}_{2} \mathrm{O}_{2}$. After 6 hours of treatment, cell survival significantly decreased when the concentration of $\mathrm{H}_{2} \mathrm{O}_{2}$ was higher than $500 \mu \mathrm{M}$ (Fig. 1a). Especially with $500 \mu \mathrm{M} \mathrm{H}_{2} \mathrm{O}_{2}$ treatment, the amount of both cleaved caspase- 3 and PARP also increased (Fig. 1b),

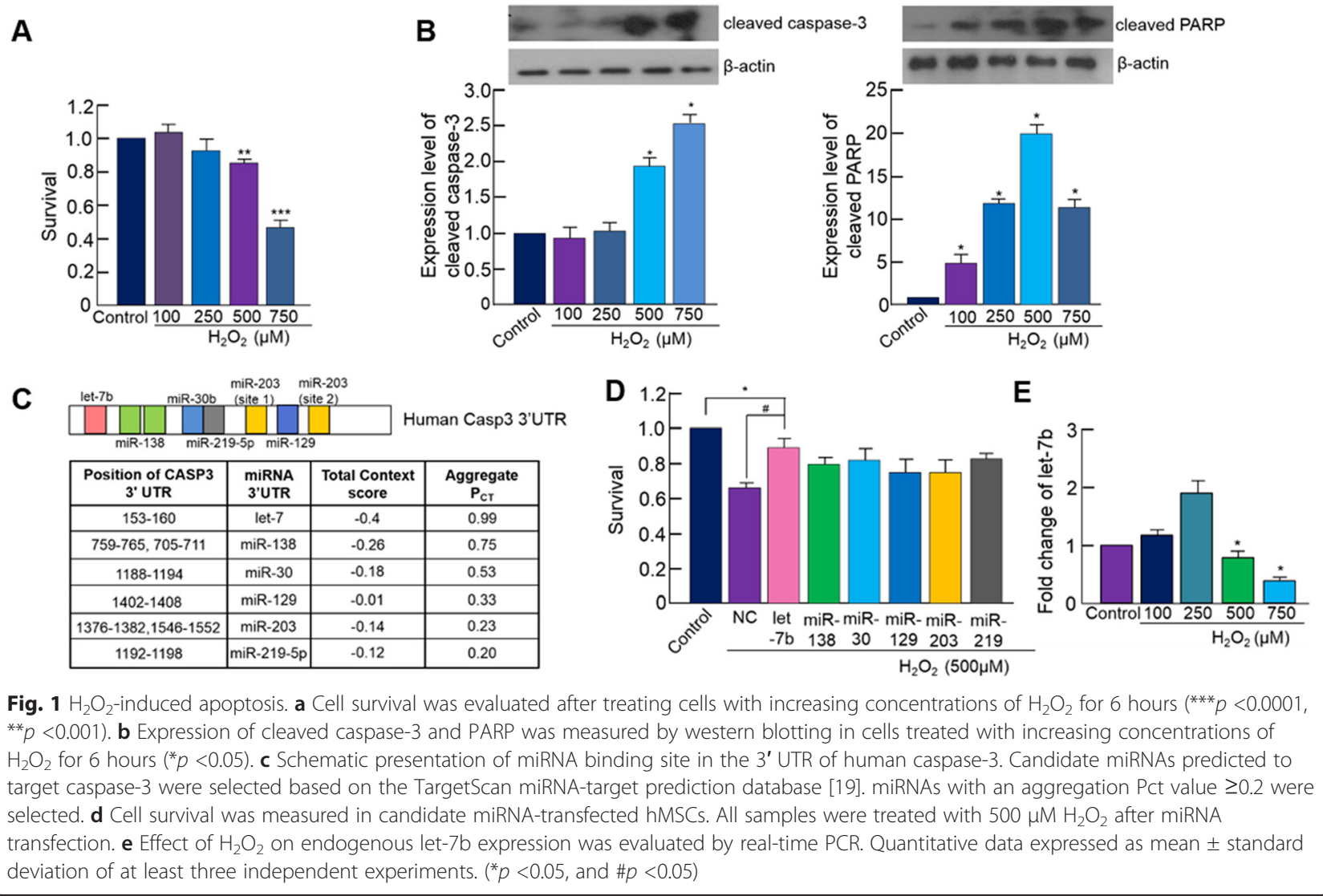


suggesting that $\mathrm{H}_{2} \mathrm{O}_{2}$ at this concentration effectively induced apoptosis hMSCs.

\section{Screening of candidate miRNAs targeting caspase-3}

To examine whether miRNA-mediated downregulation of caspase-3 can prevent $\mathrm{H} 2 \mathrm{O} 2$-mediated apoptosis, we first searched miRNAs using the TargetScan, miRNA target prediction program [19]. Six candidate miRNAs that are predicted to target caspase-3 (let-7, miR-138, miR-30b, miR-129, miR-203, and miR-219-5p) and have an aggregate Pct greater than 0.2 were selected (Fig. 1c). To empirically validate caspase- 3 targeting of those selected miRNAs, the cells were first transfected with each candidate miRNA, and then miRNA transfected cells were exposed to $500 \mu \mathrm{M}$ of $\mathrm{H}_{2} \mathrm{O}_{2}$ for 6 hours. The cell survival data after miRNA transfection and $\mathrm{H}_{2} \mathrm{O}_{2}$ treatment indicated that let-7b significantly attenuated cell survival after $\mathrm{H}_{2} \mathrm{O}_{2}$ exposure, while other candidate miRNA had no significant effect on cell survival (Fig. 1d). These data suggest that let-7b may exert anti-apoptotic effect. Additionally, $\mathrm{H}_{2} \mathrm{O}_{2}$ treatment significantly decreased the expression of endogenous let-7b at high concentrations (500 and $750 \mu \mathrm{M}$ ) (Fig. 1e), further suggesting that decreased let-7b from $\mathrm{H}_{2} \mathrm{O}_{2}$ treatment may be linked to the decreased cell survival after given concentrations of $\mathrm{H}_{2} \mathrm{O}_{2}$ treatment.

\section{Inhibition of caspase- 3 by let-7b}

Sequence alignment indicated that let- $7 \mathrm{~b}$ recognizes and binds to the sequence between 1069 and 1900 of the caspase-3 3' UTR (Fig. 2a). When the cells were transfected with $100 \mathrm{nM}$ let-7b mimics, the amount of cellular let-7b detected by real-time PCR increased approximately 40-fold compared with nontransfected cells (Fig. 2b). Transfection of let-7b mimics significantly decreased the expression of caspase-3 (Fig. 2c) and the luciferase activity of cells transfected with luciferase vector containing the $3^{\prime}$ UTR of human caspase-3 (Fig. 2d), indicating that let-7b directly targeted caspase- 3 .

\section{Visual detection of let-7b in vitro using a MB}

We utilized MBs to detect intracellular let-7b. MBs are oligonucleotide hybridization probes that indicate the presence of specific nucleic acids. The Cy3-modified longer sequence of the $\mathrm{MB}$ complementarily matches the sequence of let-7b, so that when let-7b binds to the MB it disturbs fluorophore (Cy3)-quencher (BHQ1) interaction producing fluorescence (Fig. 2e). To determine the optimal amount of $\mathrm{MB}$ for detecting let-7b, we conducted an in-vitro assay using $100 \mathrm{nM}$ let-7b mimics and increasing concentrations of $\mathrm{MB}$ designed to detect let-7b (1-100 pM), and the result indicated that $50 \mathrm{pM} \mathrm{MB}$ produced the most strong fluorescent signal (Fig. 2f). Additionally, the negative control group
(100 pM MB designed to detect miR-23 was used instead of $M B$ for let-7b) did not produce significant fluorescence with the presence of let-7b mimic, suggesting the $\mathrm{MB}$ for let-7b was indeed specific for detecting let-7b. Since denatured $M B$ should emit fluorescence, boiled $\mathrm{MB}$ (at $95{ }^{\circ} \mathrm{C}$ for 10 minutes) served as a positive control.

\section{Anti-apoptotic effect of let-7b on $\mathrm{H}_{2} \mathrm{O}_{2}$-treated MSCs}

When the cells were treated with $500 \mu \mathrm{M} \mathrm{H}_{2} \mathrm{O}_{2}$, the expression of caspase- 3 increased while let-7b expression decreased. However, such an increase of caspase- 3 and decrease of let-7b were attenuated by let-7b transfection prior to $\mathrm{H}_{2} \mathrm{O}_{2}$ treatment (Fig. 3a). To determine the effect of let-7b on survival-related signaling, we examined the phosphorylation of MEK and ERK and the expression of anti-apoptotic protein $\mathrm{Bcl}-2 . \mathrm{H}_{2} \mathrm{O}_{2}$ decreased phosphorylation of both MEK and ERK and the expression of Bcl-2. However, this $\mathrm{H}_{2} \mathrm{O}_{2}$-induced decrease was attenuated by let-7b transfection prior to $\mathrm{H}_{2} \mathrm{O}_{2}$ treatment (Fig. 3b). Furthermore, increased caspase-3 activity by $\mathrm{H}_{2} \mathrm{O}_{2}$ treatment was also attenuated by let- $7 \mathrm{~b}$ transfection (Fig. 3c), and cell survival was also recovered by let-7b transfection (Fig. 3d), indicating that let-7bmediated downregulation of caspase- 3 attenuated $\mathrm{H}_{2} \mathrm{O}_{2}$ induced cell death. To further validate the anti-apoptotic effect of let-7b, Annexin V/PI staining and TUNEL assays were performed on $\mathrm{H}_{2} \mathrm{O}_{2}$-treated cells with or without let-7b transfection prior to $\mathrm{H}_{2} \mathrm{O}_{2}$ treatment. Annexin V/PI staining indicated that $\mathrm{H}_{2} \mathrm{O}_{2}$ increased both Annexin V and PI staining of MSCs showing increased apoptosis, but this was attenuated by let-7b transfection prior to $\mathrm{H}_{2} \mathrm{O}_{2}$ treatment (Fig. 3e). Furthermore, the number of TUNEL-positive apoptotic cells increased by $\mathrm{H}_{2} \mathrm{O}_{2}$ treatment, but such an increase was suppressed by let-7b transfection (Fig. 3f), suggesting that the delivery of exogenous let-7b effectively prevented $\mathrm{H}_{2} \mathrm{O}_{2}$-induced apoptosis of MSCs. Previous studies have demonstrated that miRNAs were involved in the regulation of autophagic pathway in various cell types [22, 23]. Thus, we also examined the effect of let$7 \mathrm{~b}$ on autophagy-related genes. $\mathrm{H}_{2} \mathrm{O}_{2}$ treatment increased the expression of $\mathrm{LC} 3 \mathrm{~A} / \mathrm{B}$, indicating activation of autophagic pathway. However, such an increase of autophagic activity was attenuated by let-7b transfection prior to $\mathrm{H}_{2} \mathrm{O}_{2}$ treatment, and this was confirmed by both immunocytochemistry (Fig. $3 \mathrm{~g}$ ) and western blot (Fig. 3h). Additionally, the expression of autophagyrelated genes, such as ATG5, ATG7, ATG12, and BECN1, was attenuated in the MSCs transfected with let-7b (Fig. 3i). These results indicated that let-7b regulates the expression of autophagy-related genes at both the mRNA and protein levels. 


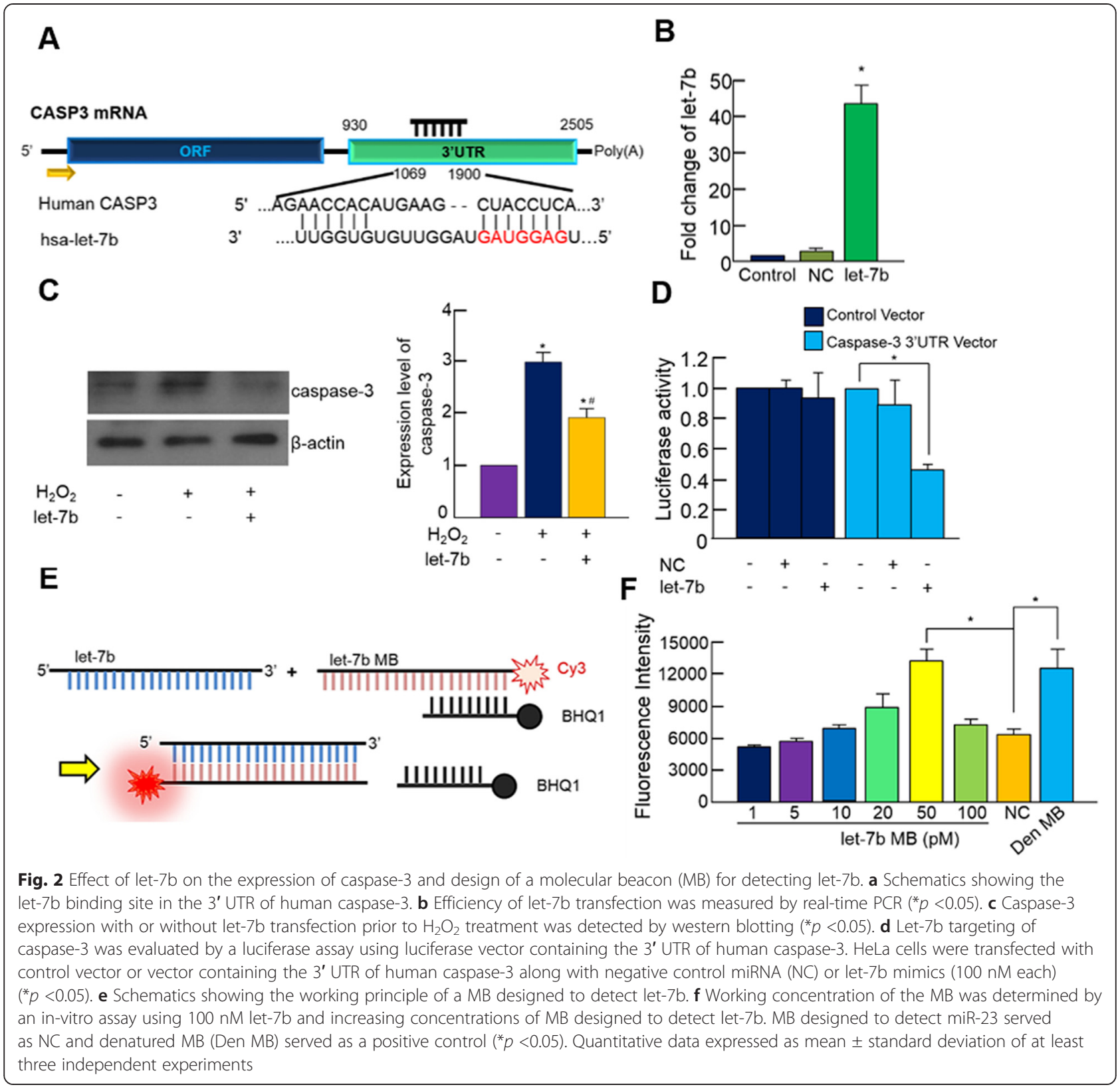

\section{Let-7b-enriched MSCs restore cardiac function after ischemic injury}

To examine the therapeutic potential of let-7b-enriched MSCs in ischemic myocardium, we transplanted let-7benriched MSCs into ischemic rat hearts. The area of fibrosis significantly decreased in the hearts transplanted with let-7b-enriched MSCs (let-7b-MSCs) compared with those of untreated ischemic hearts or the hearts transplanted with negative control miRNA-enriched MSCs (NC-MSCs) (Fig. 4a). To assess the survival of transplanted MSCs, we stained the cells with 4',6-diamidino-2-phenylindole (DAPI) prior to transplantation. The number of DAPI-positive cells was counted 3 days after the transplantation, and the results indicated that the number of DAPI-stained cells increased in the let7b-MSC transplanted heart compared with the NC-MSC transplanted heart (Fig. 4b). The result of immunohistochemistry using endothelial cell marker CD31 indicated that the transplantation of let-7b-enriched MSCs increased the number of CD31-positive cells per field in infarcted myocardium compared with NC-MSC transplanted heart (Fig. 4c), while the number of caspase3-positive cells decreased in this group (Fig. 4d), indicating that the transplantation of let-7b-enriched MSCs effectively prevented apoptosis but enhanced angiogenesis in infarcted myocardium. Additionally, analysis of cardiac 
A
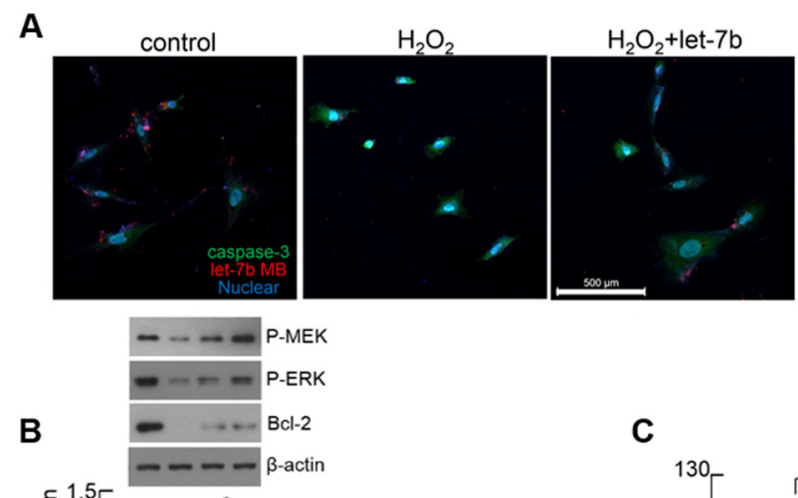

C

D
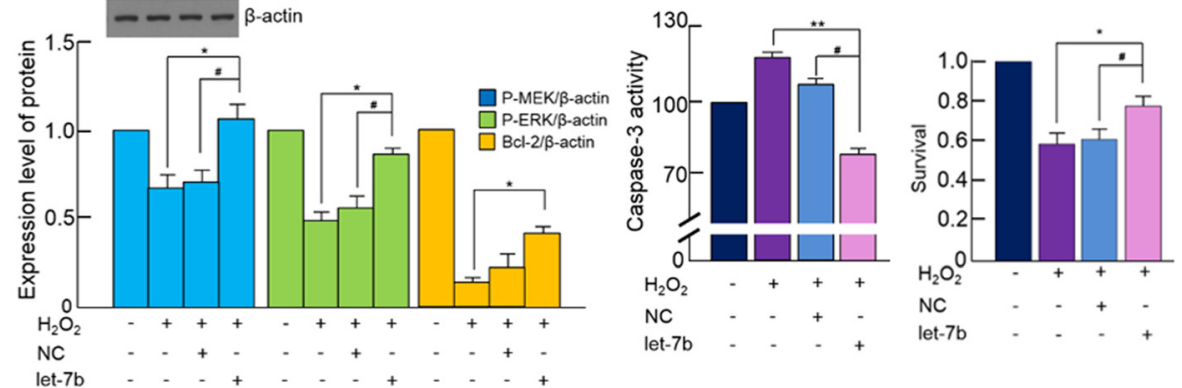

E

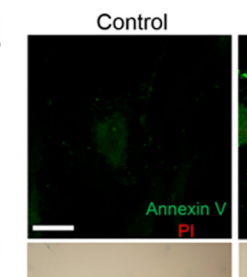

$\mathrm{H}_{2} \mathrm{O}_{2}$

$\mathrm{H}_{2} \mathrm{O}_{2}+\mathrm{NC}$

$\mathrm{H}_{2} \mathrm{O}_{2}+$ let-7b
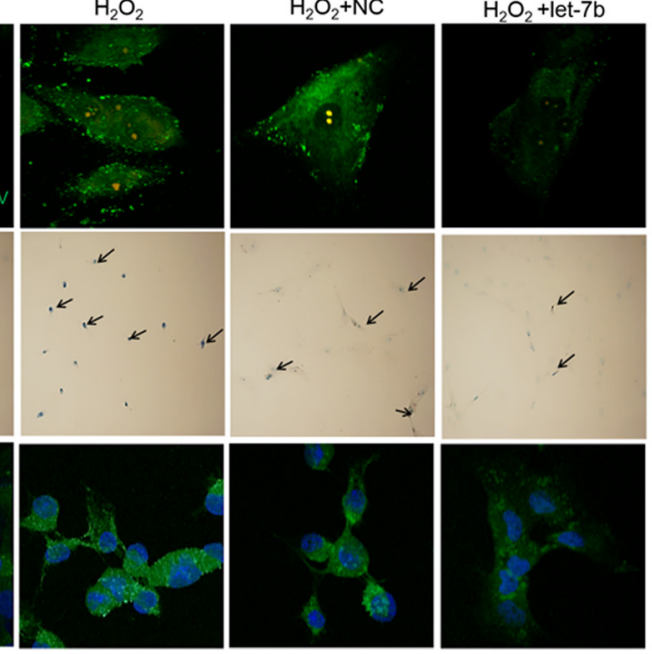

G
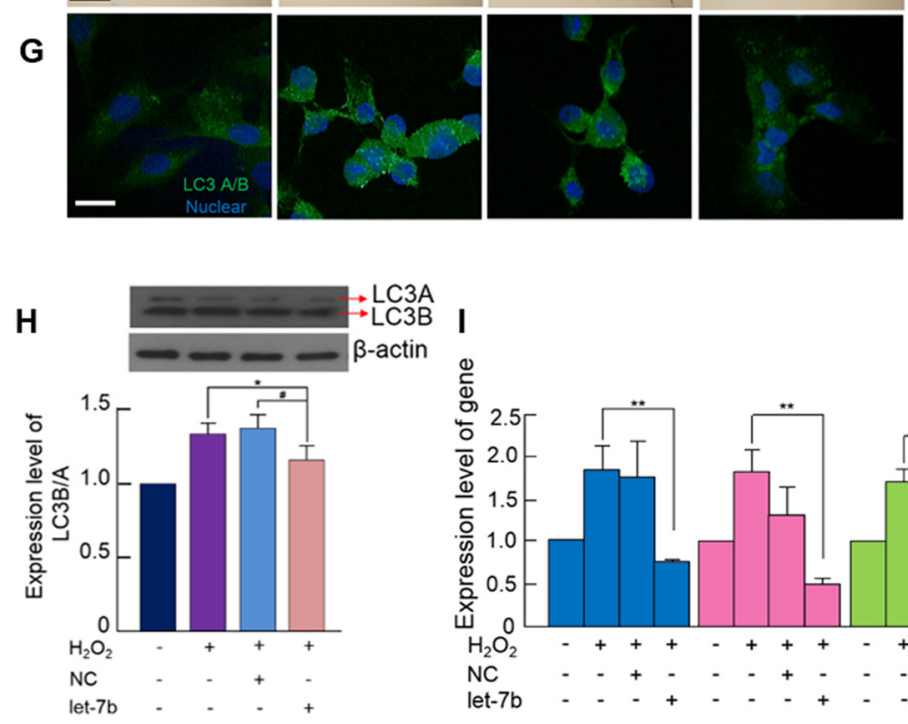

$\rightarrow$ LC3A

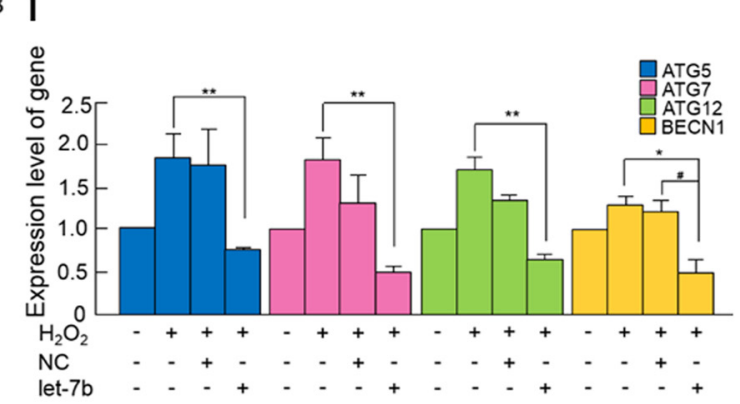

Fig. 3 (See legend on next page.) 
(See figure on previous page.)

Fig. 3 let-7b upregulates survival signaling but downregulates autophagy-related molecules. a Intracellular caspase-3 (green) and let-7b (red) were visualized under confocal microscopy. The cells were either untreated (control), $\mathrm{H}_{2} \mathrm{O}_{2}$-treated $\left(\mathrm{H}_{2} \mathrm{O}_{2}\right)$, or transfected with let-7b prior to $\mathrm{H}_{2} \mathrm{O}_{2}$ treatment $\left(\mathrm{H}_{2} \mathrm{O}_{2}+\right.$ let-7b). Nuclei stained blue. Scale bar $=500 \mu \mathrm{m}$. b Various survival-related proteins were detected by western blotting. $\left({ }^{*} p<0.05, \# p<0.05\right)$. c Caspase-3 activity and $\mathbf{d}$ cell survival was evaluated. ( ${ }^{*} p<0.05, \# p<0.05$ ). e Annexin V (green)/PI (red) staining. $\mathbf{f}$ TUNEL staining. $\mathbf{g}$ LC3A/B (green) was detected by confocal microscopy. Nuclei stained blue. Scale bar $=100 \mu \mathrm{m}$. $\mathbf{h}$ Expression of LC3A/B was detected by western blotting $\left({ }^{*} p<0.05\right.$, \#p<0.05). i Expression of autophagy-related genes was evaluated by real-time PCR $\left({ }^{*} p<0.001,{ }^{*} p<0.05\right.$, \# $\left.p<0.05\right)$. Quantitative data expressed as the mean \pm standard deviation of at least three independent experiments

function indicated that the transplantation of let-7benriched MSC improved functional parameters such as stroke volumes, end-diastolic volumes, end-systolic volumes, stroke work, stroke volume, and ejection fraction compared with other groups (Table 1). Taken together, these data suggest that the let-7b-mediated downregulation of caspase- 3 is a viable therapeutic strategy for the treatment of ischemic myocardium.

\section{Discussion}

MSCs have been used in cell-based therapy for cardiac repair and they are amenable to various pretransplant treatments including cytokines and growth factors, preconditioning, and genetic modification [24]. However, low cell survival after transplantation caused by apoptosis of injected cells due to the loss of matrix attachment in the infarcted region remains a major problem
[25]. Consequently, inhibition of apoptosis has long been emphasized in the treatment of heart disease. Apoptosis contributes to the pathogenesis of $I / R$ injury, and apoptosis-related caspase family molecules play important roles in the progression of heart disease [26]. In myocardial infarction, $\mathrm{Ca}^{2+}$ overload and $\mathrm{ROS}$ can induce apoptosis in the ischemic area [27]. In addition, several studies have reported that autophagy is upregulated in the injured heart $[6,27]$. However, it is still unclear whether autophagy plays a protective or detrimental role in the damaged heart. For example, autophagy during energy starvation under mild ischemic conditions delayed irreversible cell injuries, including apoptosis and necrosis [28], while autophagy under harsh conditions, such as I/R which we simulated using an animal model for this study, contributed to cell death via the generation of $\operatorname{ROS}[27,29]$. Additionally, several

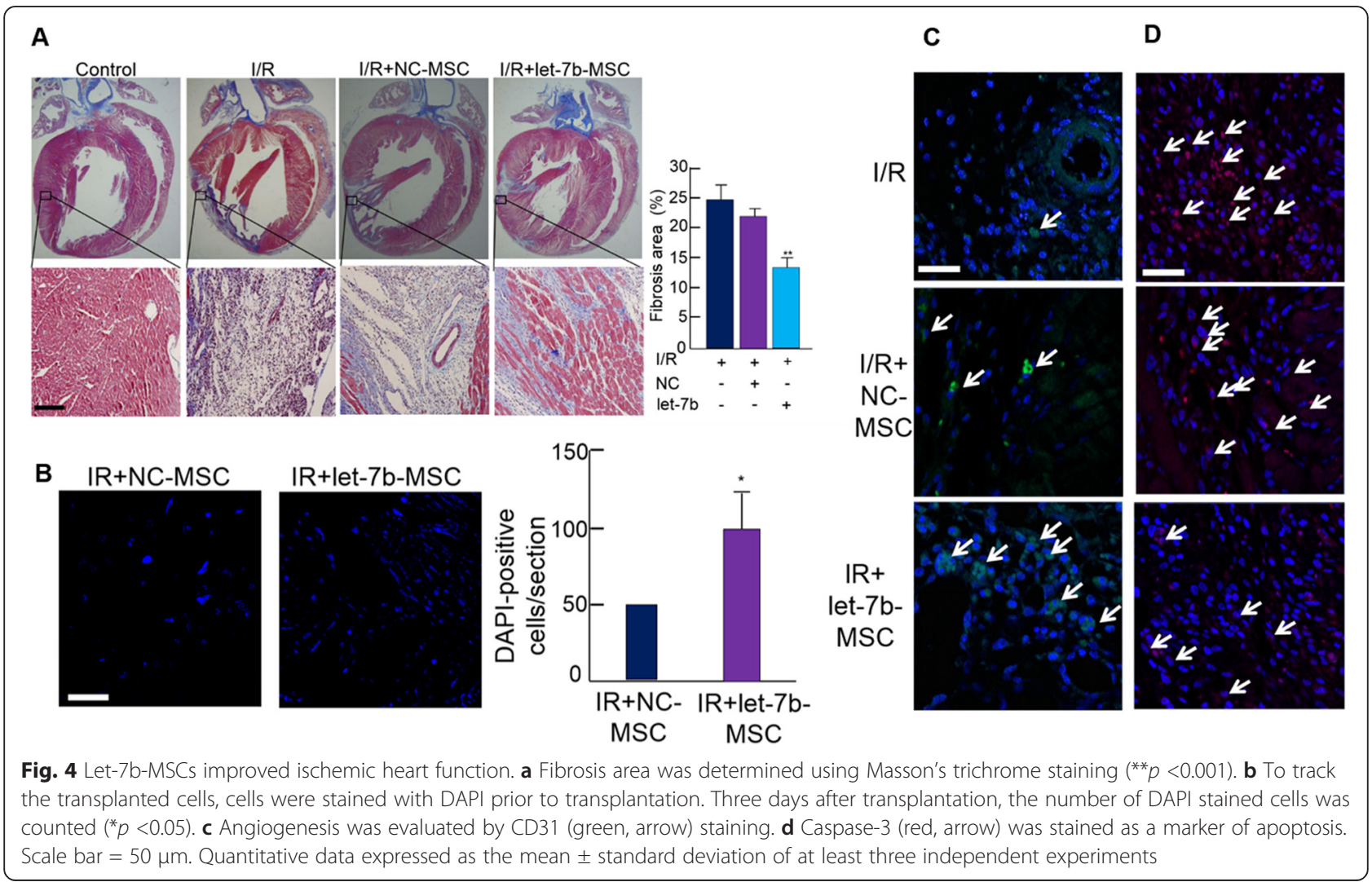


Table 1 Effect of let-7b-MSCs on cardiac function in ischemia/reperfusion animal model

\begin{tabular}{lllll}
\hline & Control & I/R & I/R + MSCs & I/R + let-7b-MSCs \\
\hline EF $(\%)$ & $69.68 \pm 1.48$ & $40.96 \pm 2.42$ & $53.4 \pm 0.92^{*}$ & $65.29 \pm 1.15^{* *}$ \\
ESV $(\mu \mathrm{l})$ & $62.67 \pm 1.82$ & $120.96 \pm 1.95$ & $87.85 \pm 1.95^{*}$ & $74.52 \pm 1.25^{* *}$ \\
EDV $(\mu \mathrm{l})$ & $124.43 \pm 1.06$ & $189.49 \pm 1.95$ & $149.48 \pm 2.17^{*}$ & $135.93 \pm 2.00^{*}$ \\
CO $(\mu \mathrm{l} /$ minute) & $37675.1 \pm 2584.66$ & $16335.9 \pm 925.71$ & $24154.9 \pm 589.04$ & $28580 \pm 500.51^{*}$ \\
SW $\left(\mathrm{mmHg}{ }^{*} \mu l\right)$ & $12710 \pm 896$ & $6335 \pm 475$ & $10245 \pm 350^{*}$ & $12526 \pm 442^{*}$ \\
SV $(\mu \mathrm{l})$ & $156.91 \pm 10.57$ & $76.9 \pm 5.79$ & $110.14 \pm 7.09^{*}$ & $112.68 \pm 1.69^{*}$ \\
\hline
\end{tabular}

All values expressed as mean \pm standard error of the mean

${ }^{*} p<0.05$ vs. I/R group

${ }^{* *} p<0.001$ vs. I/R group

CO cardiac output, EDV end-diastolic volume, EF ejection fraction, ESV end-systolic volume, $1 / R$ ischemia/reperfusion, MSC mesenchymal stem cell, SV stroke volume, SW 1:13 stroke work

published studies have suggested that crosstalk between apoptosis and autophagy exists [30]. The Atg4 family member Atg4D is cleaved by caspase-3, and the expression of beclin-1 regulates the expression of caspase- 9 $[31,32]$. In the present study, ROS increased the expressions of autophagy-related genes, including Atg5, Atg7, Atg12, and beclin-1, as well as the autophagy marker LC3A/B. However, such ROS-induced increase of autophage-related gene expressions was attenuated by let-7b transfection, and this may have attributed to the increased survival of let-7b transfected cells.

Recently, several studies have demonstrated great potential of miRNAs as a new therapeutic means for ischemic heart disease. A number of miRNAs have been reported to target caspase-3. miR-378 has been reported to attenuate apoptosis of cardiomyocytes by targeting caspase-3 [33]. Furthermore, members of the let-7 famil$\mathrm{y}-$ namely let-7a, let-7e, and let- $7 \mathrm{~g}$-have also been reported to target caspase- 3 in cancer cells, PC12 cells, and endothelial cells, respectively [26, 34, 35]. Aside from the miRNAs that are not members of the let-7 family, the predicted binding sequence of let-7 family members (a, b, c, d, e, f, g, and i) to the 3' UTR of human caspase- 3 is identical. The only difference was that let- $7 \mathrm{~b}$ had a lower context-positive score $(-0.40$ for let7b vs. -0.39 or -0.38 for other members). Since we did not examine the effect of other let-7 family members on caspase-3 expression in the present study, it is difficult to draw any conclusion on the specificity of let-7b in MSCs. This is one of the limitations of the present study, and specific roles of individual let-7 family members on the cell survival, especially of MSCs, will be an interesting subject of further study. Although additional studies are required to further elucidate the underlying mechanisms, our study also demonstrated that modulation of caspase- 3 using let-7b can be an effective means to enhance post-transplantation survival of MSCs and subsequent functional recovery of the damaged heart by upregulating survival signals such as MEK and ERK, while suppressing apoptotic signaling activation.

\section{Conclusions}

We have provided an effective strategy for enhancing cell survival after transplantation. Our study indicates that the regulation of caspase- 3 by let-7b can improve survival of hMSCs via the modulation of survival signaling and autophagy-related genes. This finding suggests that the compensation of let-7b prior to cell transplantation can be a therapeutically sound approach for treating ischemic hearts.

\section{Abbreviations}

ATG: Autophagy related; BHQ1: Black hole quencher dye 1; bp: Base pairs; Ct: Threshold cycle; DAB: 3,3'-Diaminobenzidine; DAPI: 4,6-Diamidino-2phenylindole; DMEM: Dulbecco's modified Eagle's medium; EKR: Extracellular signal regulated kinase; FBS: Fetal bovine serum; FITC: Fluorescein isothiocyanate; $\mathrm{H}_{2} \mathrm{O}_{2}$ : Hydrogen peroxide; hMSC: Human bone marrow-derived mesenchymal stem cell; I/R: Ischemia/reperfusion; LC3A/B: Light chain 3 A/B; let-7b-MSC: let-7b-overexpressing MSC; MB: Molecular beacon; MEK: Mitogenactivated protein kinase; MSC: Mesenchymal stem cell; miRNA: MicroRNA; NC: Negative control miRNA; PARP: Poly(ADP-ribose) polymerase; PBS: Phosphate-buffered saline; PI: Propidium iodide; ROS: Reactive oxygen species; TBS-T: Tris-buffered saline-0.1 \% Tween 20; TUNEL: Terminal deoxynucleotidyltransferase-mediated dUTP nick-end labeling; UTR: Untranslated region.

\section{Competing interests}

The authors declare that they have no competing interests.

\section{Authors' contributions}

$\mathrm{OH}, \mathrm{SYL}$, and $\mathrm{CYL}$ carried out the experiments, designed the study, wrote the paper, and participated in the data analysis. J-HP participated in confocal imaging. $\mathrm{JL}$ and $\mathrm{H}-\mathrm{HS}$ carried out the experiments. M-JC and EC carried out the histological analysis of hearts. SK participated in designed molecular beacon. $\mathrm{K}-\mathrm{CH}$ established the hypotheses and designed the study. All authors read and approved the manuscript.

\section{Acknowledgements}

This study was supported by a Korea Science and Engineering Foundation grant funded by the Korean government (MEST) (NRF-2011-0019243 and NRF-2011-0019254) and a grant from the Korea Health 21 R\&D Project, Ministry of Health \& Welfare, Republic of Korea (A120478).

\section{Author details}

'Catholic Kwandong University International St. Mary's Hospital, Incheon Metropolitan City 404-834, Republic of Korea. ${ }^{2}$ Department of Integrated Omics for Biomedical Sciences, Graduate School, Yonsei University, Seoul 120-752, Republic of Korea. ${ }^{3}$ Brain Korea 21 PLUS Project for Medical Science, Yonsei University College of Medicine, Seoul 120-752, Republic of Korea.

${ }^{4}$ Institute for Bio-Medical Convergence, College of Medicine, Catholic 
Kwandong University, Gangneung-si, Gangwon-do 210-701, Republic of Korea.

Received: 14 March 2015 Revised: 17 March 2015

Accepted: 17 July 2015 Published online: 22 August 2015

\section{References}

1. Huang F, Zhu X, Hu XQ, Fang ZF, Tang L, Lu XL, et al. Mesenchymal stem cells modified with miR-126 release angiogenic factors and activates Notch ligand Delta-like-4, enhancing ischemic angiogenesis and cell survival. Int J Mol Med. 2013;31:484-92.

2. Penn MS, Mangi AA. Genetic enhancement of stem cell engraftment, survival, and efficacy. Circ Res. 2008:2:1471-82.

3. Jones $E$, Yang $X$. Mesenchymal stem cells and bone regeneration: Current status. Injury. 2011;42:562-8.

4. Galle J, Bader A, Hepp P, Grill W, Fuchs B, Käs JA, et al. Mesenchymal stem cells in cartilage repair: state of the art and methods tomonitor cell growth, differentiation and cartilage regeneration. Curr Med Chem. 2011;17:2274-91.

5. Boyle AJ, McNiece IK, Hare JM. Mesenchymal stem cell therapy for cardiac repair. Methods Mol Biol. 2010;660:65-84.

6. Zhang Y, Ren J. Targeting autophagy for the therapeutic application of histone deacetylase (HDAC) inhibitors in ischemia-reperfusion heart injury. Circulation. 2014;129:1088-91.

7. Denault JB, Eckelman BP, Shin H, Pop C, Salvesen GS. Caspase 3 attenuates XIAP (X-linked inhibitor of apoptosis protein)-mediated inhibition of caspase 9. Biochem J. 2007:405:11-9.

8. Timmer JC, Salvesen GS. Caspase substrates. Cell Death Differ. 2007;14:66-72.

9. Schreiber V, Hunting D, Trucco C, Gowans B, Grunwald D, De Murcia G, et al. A dominant-negative mutant of human poly(ADP-ribose) polymerase affects cell recovery, apoptosis, and sister chromatid exchange following DNA damage. Proc Natl Acad Sci U S A. 1995;92:4753-7.

10. Chiong M, Wang ZV, Pedrozo Z, Cao DJ, Troncoso R, Ibacache M, et al. Cardiomyocyte death: mechanisms and translational implications. Cell Death Dis. 2011;2, e244.

11. Xie R, Wang F, McKeehan WL, Liu L. Autophagy enhanced by microtubule- and mitochondrion-associated MAP1S suppresses genome instability and hepatocarcinogenesis. Cancer Res. 2011;71:7537-46.

12. Panasiewicz G, Majewska M, Szafrańska B. The involvement of luteinizing hormone (LH) and pregnancy-associated glycoprotein family (PAG) in pregnancy maintenance in the pig. Reprod Biol. 2004;4:143-63.

13. Huntzinger $E$, Izaurralde $E$. Gene silencing by microRNAs: contributions of translational repression and mRNA decay. Nat Rev Genet. 2011;12:99-110.

14. Chen JF, Tao Y, Li J, Deng Z, Yan Z, Xiao X, et al. microRNA-1 and microRNA-206 regulate skeletal muscle satellite cell proliferation and differentiation by repressing Pax7. J Cell Biol. 2010;190:867-79.

15. Yu X, Cohen DM, Chen CS. miR-125b is an adhesion-regulated microRNA that protects mesenchymal stem cells from anoikis. Stem Cells. 2012;30:956-64.

16. Chang W, Lee CY, Park JH, Park MS, Maeng LS, Yoon CS, et al. Survival of hypoxic human mesenchymal stem cells is enhanced by a positive feedback loop involving miR-210 and hypoxia-inducible factor 1. J Vet Sci. 2013;14:69-76.

17. Kelly TJ, Souza AL, Clish CB, Puigserver P. A hypoxia-induced positive feedback loop promotes hypoxia-inducible factor lalpha stability through miR-210 suppression of glycerol-3-phosphate dehydrogenase 1-like. Mol Cell Biol. 2011;31:2696-706.

18. Robin TP, Smith A, McKinsey E, Reaves L, Jedlicka P, Ford HL. EWS/FLI1 regulates EYA3 in Ewing sarcoma via modulation of miRNA-708, resulting in increased cell survival and chemoresistance. Mol Cancer Res. 2012;10:1098-108.

19. TargetScan, version 6.2. www.targetscan.org.

20. Kang WJ, Cho YL, Chae JR, Lee JD, Choi KJ, Kim S. Molecular beacon-based biimaging of multiple microRNAs during myogenesis. Biomaterials. 2011:32:1915-22.

21. Song H, Cha MJ, Song BW, Kim IK, Chang W, Lim S, et al. Reactive oxygen species inhibit adhesion of mesenchymal stem cells implanted into ischemic myocardium via interference of focal adhesion complex. Stem Cells. 2010;28:555-63.

22. Matkovich SJ, Hu Y, Eschenbacher WH, Dorn LE, Dorn 2nd GW. Direct and indirect involvement of microRNA-499 in clinical and experimental cardiomyopathy. Circ Res. 2012;111:521-31.
23. Yang X, Zhong X, Tanyi JL, Shen J, Xu C, Gao P, et al. mir-30d Regulates multiple genes in the autophagy pathway and impairs autophagy process in human cancer cells. Biochem Biophys Res Commun. 2013;431:617-22.

24. Gnecchi M, Zhang Z, Ni A, Dzau VJ. Paracrine mechanisms in adult stem cell signaling and therapy. Circ Res. 2008;103:1204-19.

25. Ingber DE. Mechanical signaling and the cellular response to extracellular matrix in angiogenesis and cardiovascular physiology. Circ Res. 2002;91:877-87.

26. Peng G, Yuan Y, He Q, Wu W, Luo BY. MicroRNA let-7e regulates the expression of caspase-3 during apoptosis of PC12 cells following anoxia/ reoxygenation injury. Brain Res Bull. 2011;86:272-6.

27. Konstantinidis K, Whelan RS, Kitsis RN. Mechanisms of cell death in heart disease. Arterioscler Thromb Vasc Biol. 2012;32:1552-62.

28. Przyklenk K, Dong Y, Undyala W, Whittaker P. Autophagy as a therapeutic target for ischaemia/reperfusion injury? Concepts, controversies, and challenges. Cardiovasc Res. 2012;94:197-205.

29. Hamacher-Brady A, Brady NR, Gottlieb RA. Enhancing macroautophagy protects against ischemia/reperfusion injury in cardiac myocytes. J Biol Chem. 2006;281:29776-87.

30. Matsui Y, Takagi H, Qu X, Abdellatif M, Sakoda H, Asano T, et al. Distinct roles of autophagy in the heart during ischemia and reperfusion: roles of AMP-activated protein kinase and Beclin 1 in mediating autophagy. Circ Res. 2007;100:914-22.

31. Rubinstein $A D$, Kimchi $A$. Life in the balance-a mechanistic view of the crosstalk between autophagy and apoptosis. J Cell Sci. 2012;125:5259-68.

32. Betin VM, Lane JD. Caspase cleavage of Atg4D stimulates GABARAPL1 processing and triggers mitochondrial targeting and apoptosis. J Cell Sci. 2009;122:2554-66.

33. Fang J, Song XW, Tian J, Chen HY, Li DF, Wang JF, et al. Overexpression of microRNA-378 attenuates ischemia-induced apoptosis by inhibiting caspase-3 expression in cardiac myocytes. Apoptosis. 2012;17:410-23.

34. Tsang WP, Kwok TT. Let-7a microRNA suppresses therapeutics-induced cancer cell death by targeting caspase-3. Apoptosis. 2008;13:1215-22.

35. Zhang Y, Chen N, Zhang J, Tong Y. Hsa-let-7g miRNA targets caspase-3 and inhibits the apoptosis induced by ox-LDL in endothelial cells. Int J Mol Sci. 2013;14:22708-20

\section{Submit your next manuscript to BioMed Central and take full advantage of:}

- Convenient online submission

- Thorough peer review

- No space constraints or color figure charges

- Immediate publication on acceptance

- Inclusion in PubMed, CAS, Scopus and Google Scholar

- Research which is freely available for redistribution 